\title{
Barreiras Percebidas para a Prática de Atividade Física entre Servidores do Setor Administrativo de uma Faculdade do Distrito Federal
}

\author{
PERCEIVED BARRIERS TO THE PRACTICE OF PHYSICAL ACTIVITY AMONG SERVERS OF THE ADMINISTRATIVE \\ SECTOR OF A FACULTY OF THE FEDERAL DISTRICT
}

Luciana Zaranza Monteiro', Bruno Alves de Lira², Priscila de Souza ${ }^{3}$, Francelino D Braga Júnior ${ }^{4}$

1. Professora do Departamento de Educação Fisica do Centro Universitário do Distrito Federal (UDF), Brasilia, DF, Brasil.

2. Graduado em Educação Fisica pelo Departamento de Educação Física do Centro Universitário do Distrito Federal (UDF), Brasilia, DF, Brasil.

3. Graduada em Educação Fisica pelo Departamento de Educação Física do Centro Universitário do Distrito Federal (UDF), Brasilia, DF, Brasil.

4. Professor do Centro Universitário do Distrito Federal (UDF), Brasilia, DF, Brasil.

\begin{abstract}
RESUMO
0 objetivo deste estudo foi comparar entre os gêneros a prevalência de barreiras e sua associação com a prática de atividades físicas em servidores de uma faculdade do Distrito Federal. Trata-se de um estudo transversal com 270 servidores administrativos de uma faculdade do Distrito Federal, Brasil, desenvolvido entre outubro de 2016 a maio de 2017. Foram coletadas informações sociodemográficas, prática de atividade física e barreiras percebidas. As barreiras foram analisadas individualmente e agrupadas em domínios (ambiental, comportamental, físico e social). Associações entre barreiras percebidas (por domínios) e prática de atividades físicas foram testadas por meio de Regressão de Poisson. Resultados: As principais barreiras percebidas pelos servidores, de ambos os gêneros, foram jornada de trabalho extensa (mulheres $=33,9 \%$, homens $=24,1 \%$ ), falta de energia (mulheres $=17,3 \%$, homens $=14,9 \%$ ). As mulheres e os homens que percebiam barreiras sociais para a prática de atividade física apresentaram, probabilidade maior $(\mathrm{OR}=2,13$; IC95\% $\%=1,08-5,40$; $\mathrm{OR}=1,92$; IC95\% $\%=0,87-4,88$, respectivamente) de serem inativos fisicamente. As barreiras físicas estiveram associadas à inatividade física nas mulheres $(\mathrm{OR}=1,82$; IC95\%=1,41-4,47). As barreiras do domínio social estiveram associadas à inatividade física, para ambos os gêneros. 0 desenvolvimento de políticas públicas saudáveis com o propósito de aumentar o estímulo a prática de atividade física.
\end{abstract}

(Zaranza L, Alves B, Souza P, Braga F, 2018. Barreiras Percebidas para a Prática de Atividade Física entre Servidores do Setor Administrativo de uma Faculdade do Distrito Federal. Cienc Trab. MayAgo; 20 [62]: 97-102).

Palavras-chave: ATIVIDADE FÍSICA, TRABALHO,ESTILO DE VIDA, PREVENÇÃO.

\section{ABSTRACT}

The objective of this study was to compare the prevalence of barriers and their association with physical activity in servers of a Federal District college. It is a cross-sectional study with 270 administrative servers from a faculty of the Federal District, Brazil, developed between October 2016 and May 2017. Sociodemographic information, physical activity practice and perceived barriers were collected. The barriers were analyzed individually and grouped into domains (environmental, behavioral, physical and social). Associations between perceived barriers (by domains) and practice of physical activities were tested by means of Poisson Regression. Results: The main barriers perceived by the employees of both genders were extensive work hours (women $=33.9 \%$, men $=24.1 \%$ ), lack of energy (women $=17.3 \%$, men $=14.9 \%$ ). Women and men who perceived social barriers to physical activity presented a higher probability $(\mathrm{OR}=2.13,95 \% \mathrm{CI}=1.08-5.40, \mathrm{OR}=1.92,95 \% \mathrm{CI}=0.87,4.88$, respectively) of being physically inactive. Physical barriers were associated with physical inactivity in women $(\mathrm{OR}=1.82,95 \% \mathrm{CI}=$ 1.41-4.47). The barriers of the social domain were associated with physical inactivity for both genders. The development of healthy public policies with the purpose of increasing the stimulus to the practice of physical activity.

Key words: PHYSICAL ACTIVITY, JOB, LIFESTYLE, PREVENTION.
Correspondencia / Correspondence:

Profa. Dra. Luciana Zaranza Monteiro

Centro Universitário do Distrito Federal - UDF - Escola da Saúde

SEP/SUL EQ 704/904 Conj. A. Bairro: Asa Sul. Tel: 61 3704-8859

CEP: 70390-045 - Brasilia-DF-Brasil.

e-mail: lucianazaranza@hotmail.com

Recibido: 19 de Marzo de 2018 / Aceptado: 25 de Julio de 2018

\section{INTRODUÇÃO}

0 século passado foi caracterizado por mudanças profundas no estilo de vida dos seres humanos e pelos avanços científicos na área da saúde. Incentivado pela industrialização, robotização e informatização dos meios de produção, o homem tornou-se um ser dependente de novas práticas cotidianas que dispensam o modo primitivo de viver dos seus antepassados. ${ }^{1}$ Dentre os reflexos dessas novas práticas, destaca-se a adoção de um estilo de vida mais sedentário, resultado das mudanças ocorridas na dinâmica populacional 
através do processo de transição demográfica, modernização dos meios de transporte, facilidade na obtenção de alimentos e inserção de máquinas e computadores nas tarefas cotidianas e laborais. ${ }^{1,2}$

Nesse contexto, a prevalência de comportamentos de risco no local de trabalho, como o sedentarismo, vem aumentando gradativamente a cada ano, principalmente nos países desenvolvidos e em desenvolvimento, e que aliados a fatores comportamentais como o consumo de bebidas alcóolicas, tabaco e dieta inadequada, formam os principais fatores associados ao risco de doenças cardiovasculares. ${ }^{2}$

0 local de trabalho tem sido foco de pesquisas sobre inatividade física, visto que baixos níveis de atividade física influenciam negativamente à saúde do trabalhador. Indivíduos que não atendem as recomendações da prática de atividade física (AF) são mais suscetíveis ao absenteísmo e afastamento do trabalho por desenvolvimento de doenças, são mais propensos a desenvolver distúrbios ocupacionais, como lesões por esforço repetitivo; apresentam maiores níveis de estresse e indisposição para realização de tarefas coridianas. ${ }^{3,4}$

De acordo com as recomendações da prática de atividade física para adultos, para adquirir benefícios à saúde os indivíduos devem realizar pelo menos 150 minutos semanais de atividade em intensidade moderada ou 75 minutos de atividade vigorosa. ${ }^{5}$ Contudo, dados disponíveis sugerem que $31 \%$ da população adulta mundial não atende as recomendações mínimas para a prática de atividade física. ${ }^{6}$ Estudos realizados com servidores públicos encontraram prevalência de baixo nível de atividade física de 49,4\% no estado da Bahia ${ }^{7}$ e 56,7\% no sul do Brasil. ${ }^{8}$ Ademais, têm sido investigado os possíveis fatores correlatos ao baixo nível de atividade física de trabalhadores. ${ }^{9}$ Mulheres demonstraram ser menos ativas quando comparadas aos homens, entretanto, para as variáveis sociodemográficas como a idade, a escolaridade e o nível econômico, os resultados apresentamse controversos. ${ }^{10}$

Baixos niveis de atividade física também preocupam os órgãos de saúde, porque é fator de risco para várias doenças crônicas não transmissíveis (DCNT), como, por exemplo, o diabetes mellitus tipo 2, hipertensão arterial, hipercolesterolemia. ${ }^{10}$

Com escassas evidências na literatura brasileira e as barreiras sendo fortes determinantes na participação de atividades físicas, é importante a realização de estudos com funcionários que atuam nas instituições de ensino, principalmente no setor administrativo, limpeza e segurança, a fim de procurar elucidar quais as principais barreiras para a prática de atividade física e a partir destas informações contribuir com recomendações para o aumento de comportamentos fisicamente ativos na determinada população.

Diante do exposto, este estudo objetivou comparar entre os gêneros a prevalência de barreiras e sua associação com a prática de atividades físicas em servidores de uma faculdade do Distrito Federal, DF, Brasil.

\section{MATERIAIS E MÉTODOS}

Trata-se de um estudo transversal, realizado, entre outubro de 2016 a maio de 2017, com servidores em efetivo exercício profissional do setor administrativo de uma Faculdade no Distrito Federal.

\section{Participantes do estudo}

0 cálculo amostral levou em consideração a quantidade de funcionários no setor administrativo. Foi adotado erro tolerável de cinco pontos percentuais, nível de confiança de 95\% e uma prevalência desconhecida para o desfecho (50\%). Dessa forma, os participantes deste estudo foram selecionados por meio de uma amostragem por conveniência ( $\mathrm{n}=270$ servidores, de um total de 345 ).

Todos os participantes foram convidados a participar da pesquisa. Foram incluídos no estudo os funcionários da faculdade que aceitaram participar e assinaram o Termo de Consentimento Livre e Esclarecido (TCLE).

\section{Procedimentos para coleta dos dados e instrumentos de pesquisa}

Os pesquisadores explicaram aos funcionários presentes os objetivos e a importância do estudo e convidou a todos para responderem aos questionários, compostos por questões sociodemográficas, sobre a prática de atividade física e as barreiras percebidas para a mesma.

A respeito das questões sociodemográficas, os participantes informaram o seu sexo (masculino, feminino), idade (20-39 anos, 40-49 anos, 50 e mais anos), estado civil foi categorizado em "sem companheiro" (solteiro, separado ou viúvo) e "com companheiro" (casado), nível de escolaridade (ensino médio completo, ensino superior incompleto, ensino superior completo), tempo (anos) de serviço na faculdade ( $<1$ ano, 1 a 5 anos, 6 a 10 anos, $\geq 11$ anos), carga horária de trabalho semanal ( $<40$ horas e $\geq 40$ horas). 0 nivel socioeconômico foi estimado pelo questionário da Associação Brasileira de empresas de pesquisa - 2016, que divide a população brasileira em 5 classes econômicas, por ordem decrescente de poder de compra (A1, A2, B1, B2, C1, $\mathrm{C} 2$, D e E). No presente estudo, as categorias foram dicotomizadas em classe alta (A1; A2; B1; B2) e classe baixa (C1; C2; D; E).

A variável dependente foi o nível de atividade física. 0 Questionário Internacional de Atividade Física (IPAQ) versão curta ${ }^{11}$ foi empregado para analisar essa variável. Este questionário é composto por oito questões e tem como objetivo fazer com que o entrevistado relate as atividades físicas desenvolvidas em uma semana anterior, classificando-as em relação à quantidade de atividades físicas realizadas. Cinco categorias são geradas de acordo com as respostas relatadas: muito ativo, ativo, irregularmente ativo $\mathrm{A}$, irregularmente ativo B, sedentário. Sendo que o critério de recomendação para a prática de atividade física é de frequência mínima de cinco dias na semana, ou duração mínima de 150 minutos por semana. As cinco categorias do instrumento IPAQ foram dicotomizadas neste estudo, passando a serem classificadas como: insuficientemente ativos - para os indivíduos que obtiveram os níveis de atividade física de "irregularmente ativos A, B e sedentários"; e ativos - para os indivíduos que obtiveram os níveis de atividade física de "muito ativos e ativos".

As barreiras percebidas para a prática de atividade física foram identificadas por meio de um questionário proposto para indivíduos adultos e validado previamente por Martins e Petroski. ${ }^{12}$ No instrumento são apresentados 19 motivos que possivelmente bloqueiam ou impedem a realização de $\mathrm{AF}$, cujas respostas são obtidas por meio de escala likert ( 1 = nunca; 2 = raramente; 3 = às vezes; 4 = quase sempre e 5 = sempre). Considerou-se como barreira percebida os valores correspondentes as respostas " 4 e 5 "e barreira ausente os valores "1,2 e 3 ". ${ }^{13}$ A prevalência das 19 barreiras foi analisada e, posteriormente, as mesmas foram agrupadas e analisadas em quatro domínios principais: domínio ambiental, social, comportamental e físico, conforme categorização utilizada em estudo prévio. ${ }^{14}$

A variável nutricional foi definida através do cálculo do Índice de Massa Corporal (IMC) obtido pela divisão do peso (em quilogramas) pelo quadrado da altura (em metros). Para o cálculo, utilizou-se peso e altura auto-referidos e pontos de corte que classificaram os indivíduos nas seguintes categorias: baixo peso $(<18,5)$, normal $(18,5$ a $24,9)$, sobrepeso (25 a 29,9$)$ e obesidade $(\geq 30) .{ }^{15}$ 


\section{Análise dos dados}

Os dados foram duplamente digitados e checados em relação a possíveis inconsistências. Realizou-se análise descritiva dos dados para verificar frequências, bem como médias e desvio padrão. Utilizou-se o teste Qui-quadrado e o teste exato de Fisher para comparar prevalências. Utilizou-se a regressão de Poisson para verificar possiveis associações entre os domínios das barreiras para a prática de AF.

$\mathrm{Na}$ análise ajustada foram incluídos todos os domínios das barreiras, além do sexo, idade e nível socioeconômico dos servidores. Todas as análises foram realizadas no programa STATA 12.0, e o nível de significância adotado foi de 5\%.

\section{Aspectos éticos}

0 estudo seguiu as diretrizes da Resolução n 466/2012 do Conselho Nacional de Saúde e foi aprovado pelo Comitê de Ética em pesquisa do Centro Universitário do Distrito Federal - UDF, recebendo aprovação (CAAE: 55019116.3.0000.5650).

\section{RESULTADOS}

As características gerais dos participantes estão apresentadas na Tabela 1. A maior parte da amostra foi constituída por indivíduos do sexo feminino (60\%), com idade de 40 a 49 anos (48,5\%), que estavam com companheiro (61,2\%), trabalhavam de 5 a 10 anos na faculdade (37,4\%), com carga horária de trabalho semanal $\geq 40$ horas $(60,8 \%)$, apresentavam nível econômico baixo $(65,9 \%)$, a maioria

Tabela 1.

Descrição da amostra segundo características dos servidores administrativos.

\begin{tabular}{|c|c|c|}
\hline Variável & $n$ & $\%$ \\
\hline \multicolumn{3}{|l|}{ Sexo } \\
\hline Feminino & 162 & 60 \\
\hline Masculino & 108 & 40 \\
\hline \multicolumn{3}{|l|}{ Idade (anos) } \\
\hline 20 a 39 & 98 & 36,3 \\
\hline 40 a 49 & 131 & 48,5 \\
\hline$\geq 50$ & 41 & 15,2 \\
\hline \multicolumn{3}{|l|}{ Estado Civil } \\
\hline Com companheiro (a) & 167 & 61,2 \\
\hline Sem companheiro (a) & 103 & 38,8 \\
\hline \multicolumn{3}{|c|}{ Nivel de escolaridade (último nivel) } \\
\hline Ensino médio completo & 72 & 26,7 \\
\hline Ensino superior incompleto & 136 & 50,3 \\
\hline Ensino superior completo & 62 & 23 \\
\hline \multicolumn{3}{|l|}{ Tempo de serviço (anos) } \\
\hline$<1$ & 28 & 10,3 \\
\hline 1 a 5 & 87 & 32,3 \\
\hline 5 a 10 & 101 & 37,4 \\
\hline$\geq 11$ & 54 & 20 \\
\hline \multicolumn{3}{|l|}{ Carga horária semanal } \\
\hline$<40$ horas & 106 & 39,2 \\
\hline$\geq 40$ horas & 164 & 60,8 \\
\hline \multicolumn{3}{|l|}{ Nivel econômico } \\
\hline Classe alta & 92 & 34,1 \\
\hline Classe baixa & 178 & 65,9 \\
\hline \multicolumn{3}{|l|}{ Estado nutricional (kg/m2) } \\
\hline Normal & 54 & 20 \\
\hline Sobrepeso & 182 & 67,4 \\
\hline Obesidade & 34 & 12,6 \\
\hline \multicolumn{3}{|c|}{ Realiza Atividade Física ( $\geq 150 \mathrm{~min} / \mathrm{semana}$ ) } \\
\hline Sim & 82 & 30,3 \\
\hline Não & 188 & 69,7 \\
\hline Total & 270 & 100 \\
\hline
\end{tabular}

Figura 1.

Nível de Atividade Física dos servidores por sexo.

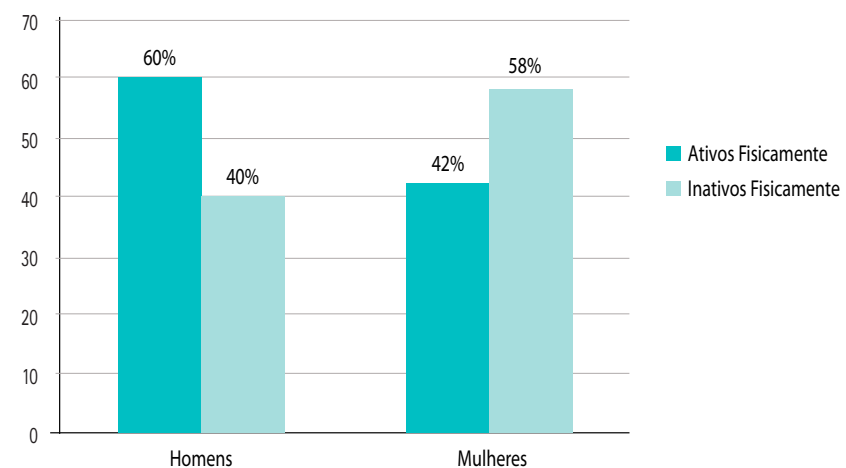

apresentou sobrepeso $(67,4 \%)$ e não realizavam atividade física de acordo com as recomendações (69,7\%).

Observou-se que a prevalência de inatividade física entre os servidores administrativos foi maior entre as mulheres (58\%) quando comparado aos homens $(40 \%)(\mathrm{p}=0,004)$ (Figura 1).

As barreiras percebidas com maior frequência pelos servidores do sexo feminino foram jornada de trabalho extensa (33,9\%), tarefas domésticas $(19,1 \%)$ e falta de energia $(17,3 \%)$, enquanto os homens alegaram a jornada de trabalho extensa $(24,1 \%)$, falta de recursos financeiros $(17,6 \%)$ e falta de energia $(14,9 \%)$ (Tabela 2$)$. Houve associação entre o gênero feminino com jornada de trabalha $(p=0,005)$ e falta de energia $(\mathrm{p}=0,004)$.

\section{Tabela 2.}

Barreiras percebidas para a prática de atividades fisicas em servidores por sexo. Brasília, DF, 2017.

\begin{tabular}{|c|c|c|c|c|c|}
\hline \multirow[t]{2}{*}{ Barreiras } & \multicolumn{2}{|c|}{ Mulheres } & \multicolumn{2}{|c|}{ Homens } & \multirow[b]{2}{*}{ p-valor } \\
\hline & $\mathrm{N}$ & $\%$ & $\mathrm{~N}$ & $\%$ & \\
\hline \multicolumn{6}{|l|}{ Dominio ambiental } \\
\hline Falta de clima adequado* & 10 & 6,2 & 8 & 7,4 & 0,824 \\
\hline \multicolumn{6}{|l|}{ Falta de espaço disponivel } \\
\hline para a prática** & 7 & 4,3 & 4 & 3,7 & 0,747 \\
\hline Falta de equipamento disponivel ${ }^{* *}$ & 14 & 8,7 & 10 & 9,2 & 0,782 \\
\hline Ambiente insuficientemente seguro** & 8 & 4,9 & 5 & 4,6 & 0,911 \\
\hline \multicolumn{6}{|l|}{ Dominio social } \\
\hline Jornada de trabalho extensa* & 55 & 33,9 & 26 & 24,1 & 0,005 \\
\hline Compromissos familiares* & 18 & 11,1 & 11 & 10,2 & 0,869 \\
\hline Tarefas domésticas ${ }^{* *}$ & 31 & 19,1 & 8 & 7,4 & 0,679 \\
\hline Falta de companhia** & 22 & 13,5 & 12 & 11,1 & 0,589 \\
\hline \multicolumn{6}{|l|}{ Falta de incentivo de familiares } \\
\hline e/ou amigos** & 12 & 7,4 & 7 & 6,5 & 0,832 \\
\hline Falta de recursos financeiros* & 16 & 9,9 & 19 & 17,6 & 0,432 \\
\hline \multicolumn{6}{|l|}{ Falta de conhecimento ou } \\
\hline \multicolumn{6}{|l|}{ Domínio comportamental } \\
\hline Mau humor** & 9 & 5,5 & 5 & 4,6 & 0,475 \\
\hline Medo de lesionar-se ${ }^{*}$ & 7 & 4,3 & 4 & 3,7 & 0,817 \\
\hline \multicolumn{6}{|l|}{ Preocupação com a aparência } \\
\hline durante a prática** & 19 & 11,7 & 14 & 13,1 & 0,436 \\
\hline Falta de interesse em praticar* & 9 & 5,5 & 7 & 6,5 & 0,728 \\
\hline \multicolumn{6}{|l|}{ Domínio físico } \\
\hline Limitações físicas ${ }^{* *}$ & 8 & 4,9 & 5 & 4,6 & 0,976 \\
\hline Dores leves ou mal estar* & 14 & 8,7 & 9 & 8,3 & 0,894 \\
\hline Falta de energia** & 28 & 17,3 & 16 & 14,9 & 0,004 \\
\hline Falta de habilidade física** & 14 & 8,7 & 8 & 7,4 & 0,653 \\
\hline
\end{tabular}


Tabela 3.

Prevalência e razões de prevalência de inatividade física, de acordo com as barreiras percebidas em servidores.

\begin{tabular}{|c|c|c|c|c|c|c|c|c|}
\hline Barreiras & RP Bruto & IC 95\% & RP* Ajustado & IC 95\% & RP Bruto & IC 95\% & RP* Ajustado & IC 95\% \\
\hline \multicolumn{9}{|c|}{ Ambientais } \\
\hline Ausência & 1,00 & & 1,00 & & 1,00 & & 1,00 & \\
\hline Presença & 1,28 & $0,74-1,97$ & 1,10 & $0,56-2,08$ & 1,05 & $0,35-1,98$ & 0,71 & $0,10-2.48$ \\
\hline Ausência & 1,00 & & 1,00 & & 1,00 & & 1,00 & \\
\hline Presença & 2,95 & $1,72-4,26$ & 2,13 & $1,08-5,40$ & 2,28 & $1,31-3,42$ & 1,92 & $0,87-4,88$ \\
\hline \multicolumn{9}{|c|}{ Comportamentais } \\
\hline Ausência & 1,00 & & 1,00 & & 1,00 & & 1,00 & \\
\hline Presença & 2,17 & $1,74-3,22$ & 1,82 & $1,41-4,47$ & 1,20 & $0,38-2,10$ & 0,78 & $0,14-1,75$ \\
\hline
\end{tabular}

RP = Razão de Prevalência; IC95\% = Intervalo de Confiança de 95\%; *Ajustada por todas as barreiras mais o sexo, idade e nível econômico.

A Tabela 3 apresenta as análises de associação entre as barreiras percebidas para a prática de AF e inatividade física nos servidores por gênero. Após ajuste por todas as barreiras, sexo, idade e nível econômico, as barreiras sociais estiveram associadas à inatividade física em ambos os gêneros, enquanto que a barreira física associou-se apenas no gênero feminino. As mulheres como os homens que percebiam barreiras sociais para a prática de $\mathrm{AF}$ apresentaram, probabilidade maior $(\mathrm{OR}=2,13$; IC95\%=1,08-5,40; $\mathrm{OR}=1,92$; IC95\%=0,87-4,88, respectivamente) de serem inativos fisicamente. As barreiras físicas estiveram associadas à inatividade física nas mulheres $(\mathrm{OR}=1,82$; IC95\%=1,41-4,47).

\section{DISCUSSÃO}

A prevalência de inatividade física foi maior nas mulheres (58\%), esses dados corroboraram com um estudo onde a prevalência foi maior nas mulheres com dependentes no domicílio (filhos e/ou pessoas doentes), relacionando-se aos papéis históricos desempenhados pelas mulheres de esposa, mãe e cuidadora, corroborando com os achados de outro estudo, no qual a presença elevada de crianças no domicílio, dificultou a prática de atividade física quando não tinham com quem deixar os filhos. ${ }^{16}$ Outra investigação mostrou que obrigações familiares e renda foram associadas à inatividade física no conjunto da população $(45,4 \%)$ e, para as mulheres, tais obrigações apresentaram significância estatística. ${ }^{17}$

Prevalências semelhantes foram apresentadas por outros autores. ${ }^{18}$ Pesquisa realizada no ano de 2002, com 704 funcionários adultos da Universidade de Brasília, identificou que 48,3\% dos servidores não praticavam nenhum tipo de atividade física durante a semana. ${ }^{8}$ Estudo transversal de base populacional, realizado em Senegal com trabalhadores adultos, relatou prevalência de inatividade física de 43,3\%. ${ }^{19}$

A diferença na prática de AF entre os sexos também foi constatada em outros estudos nacionais, como o de Florindo e colaboradores $^{20}$, cujos resultados apontaram que na idade adulta, os homens eram mais ativos que as mulheres. Outros estudos também encontraram que os homens eram mais ativos. ${ }^{21} \mathrm{~A}$ diferença de práticas de AF entre os sexos pode ser parcialmente explicada pelo desempenho da mulher nos cuidados da casa e da família, o que pode dificultar a prática de outras atividades físicas. $^{22}$

Através do IPAQ, encontramos que 30,3\% dos indivíduos eram fisicamente ativos. Outros estudos conduzidos no Brasil e que também utilizaram o IPAQ com população de servidores de universidades públicas, mostraram que, na Universidade Estadual da Bahia, 50,6\% dos sujeitos eram ativos fisicamente ${ }^{7}$ e na Universidade Estadual do Piauí, 53,6\% dos funcionários dos setores administrativos eram moderadamente ativos e 13,9\% eram muito ativos. $^{23}$

Cada vez mais, as pessoas realizam atividades ocupacionais que demandam menor esforço físico e também se locomovem com meios de transporte que não requerem grande gasto de energia, o que acarreta altas taxas de inatividade física. ${ }^{2}$ Além do desenvolvimento de DCNT, a prática insuficiente de AF pode aumentar as chances de o individuo desenvolver osteoporose, câncer, depressão, ansiedade, dentre diversos outros agravos à saúde. Para tanto, é importante que os servidores recebam orientações com intuito de incentivar a prática regular de atividade física, eis que a inatividade física aumenta de $20-30 \%$ o risco de morte precoce. ${ }^{10}$

A prática de $\mathrm{AF}$ no trabalho e no tempo livre de uma amostra representativa de trabalhadores da Holanda, e encontraram que apenas 45,3\% dos trabalhadores estavam com altos niveis de atividade física e que na média as atividades físicas ocupacionais contribuíram em 30\%. ${ }^{24}$ Já no Rio Grande, no estado do Rio Grande do Sul, verificou-se que apenas 19,5\% dos trabalhadores portuários avulsos eram insuficientemente ativos. ${ }^{25}$ No Vale do Jequitinhonha, Minas Gerais, a prevalência de trabalhadores que não praticavam 150 minutos de atividade física por semana foi de $17,1 \% .^{25}$

A prática de atividade física em trabalhadores da indústria do estado do Rio Grande do Sul e identificaram no seu estudo as barreiras percebidas pelos trabalhadores que os impedem ou dificultam a prática de $\mathrm{AF}^{2}$ Prevalência de sedentarismo foi de 45,4\%. As respostas mais prevalente para justificar a não realização de atividades físicas foram cansaço $(15,1 \%)$, seguido de excesso de trabalho (12,7\%) e obrigações familiares (9,2\%). ${ }^{2}$ Outro estudo no Distrito Federal verificou que 56\% dos trabalhadores da indústria eram poucos ativos fisicamente.9 Na Austrália, essa prevalência foi de $53 \% .^{26}$

Em relação ao estado nutricional dos servidores, nosso estudo identificou que $67,4 \%$ estão com sobrepeso, corroborando com outros estudos que também apresentam uma elevada prevalência. ${ }^{27} \mathrm{~A}$ média das capitais brasileiras é de 52,5\% de adultos com excesso de peso. ${ }^{28}$ Nos servidores da Universidade Federal de Santa Catarina, 63,6\% dos funcionários estavam com excesso de peso $^{29}$ e os servidores de uma universidade do Rio de Janeiro verificou que 59,7\% apresentavam excess de peso. ${ }^{30}$ 
Resultados semelhantes foram encontrados em estudos realizados em nipo-brasileiros residents em Bauru-SP; ${ }^{31}$ funcionários do setor administrativo de uma indústria processadora de madeiras da cidade de Botocatu-SP. ${ }^{32}$

Comparado a outros países, a prevalência de excesso de peso observada nesse estudo são inferiores às verificadas em Porto Rico $(78,4 \%)^{33}$, no Kuwait $(77,3 \%)^{34}$, e nos Estados Unidos (72,3\%). ${ }^{35}$

A elevada prevalência de excesso de peso observada entre os servidores do setor administrativo em nosso estudo deve ser analisada com atenção pelos departamentos que cuidam da saúde dos servidores, devido ao grande impacto que causam à saúde dos indivíduos. ${ }^{4} 0$ excesso de peso está relacionado a desordens cardiovasculares, metabólicas, entre outras doenças, bem como a mortalidade. ${ }^{36}$

A falta de tempo e a jornada de trabalho - ou de estudos - são motivos frequentemente apontados em estudos científicos, como principais coibentes da adesão à prática de atividades físicas, entre indivíduos de diversas faixas etárias e categorias profissionais incluindo praticantes regulares de atividades físicas. ${ }^{37}$

No atual estudo, quanto às barreiras para a prática de atividades físicas, os resultados revelaram a jornada de trabalho como o principal motivo percebido como barreira para a prática de atividades físicas entre os servidores, seguido da falta de energia, tarefas domésticas e da falta de recursos financeiros. ${ }^{13} 0$ cansaço físico pode, muitas vezes, refletir em uma percepção de dores físicas, tornando-se, portanto, um fator de impedimento para a realização de atividades físicas. ${ }^{38}$ Com base nesse raciocínio, parece fazer sentido que a jornada de trabalho extensa, barreira mais citada pelos servidores do presente estudo reflita na percepção da falta de energia. Isso pode gerar sensação de cansaço físico e dores físicas levando os universitários a procurar o descanso em seu tempo livre.

Esse resultado foi semelhante ao encontrado entre professores universitários da Universidade Federal de Santa Catarina. ${ }^{39}$ Entre outras barreiras percebidas por esses professores, destacaram-se a falta de interesse, as tarefas domésticas, a falta de companhia e a jornada de trabalho. Em um outro estudo, a falta de dinheiro foi a barreira mais frequente $(40,3 \%)$, seguida pelo cansaço $(38,1 \%)$, falta de companhia $(32,2 \%)$ e de tempo $(31,5 \%) .{ }^{40}$

Entre os policiais de Feira de Santana, a jornada de trabalho foi o segundo motivo mais frequente percebido como barreira para a prática de atividades físicas. ${ }^{24}$ Isto pode estar relacionado com a baixa remuneração, que é uma situação que obriga os policiais a terem outros empregos informais; e os professores universitários a darem aulas em mais do que uma Instituição de Ensino Superior, o que origina sua falta de tempo para atividades físicas, por conta dos compromissos de trabalho.
Outra observação importante é que uma jornada de trabalho extensa pode influenciar os indivíduos a perceberem como barreira para a prática de atividades físicas, motivos como a falta de energia, a ocorrência de dores e mal estar, o mau humor, em função da exigência física e psicológica da atividade laboral apresentada como altamente estressante. Mas é possível, também, que os compromissos familiares sejam influenciados da mesma maneira, já que o fato de o indivíduo ter pouco tempo livre devido ao trabalho pode leva-lo a optar por ficar em casa, na companhia de sua família, o que termina dificultando a prática de uma atividade física.

Observou-se que, entre os servidores investigados, os compromissos familiares e as tarefas domésticas foram mais frequentes entre as mulheres. Esse resultado ilustra questões relacionadas ao, ainda predominante, papel de cuidadora da família e de principal executora das tarefas domésticas, atribuído à mulher. ${ }^{19}$

A respeito das análises de associação, verificou-se que os servidores, de ambos os gêneros, que percebiam barreiras sociais para a prática de AF apresentaram maior probabilidade de serem inativos fisicamente. É importante destacar que o domínio social inclui fatores relacionados a "jornada de trabalho extensa", "tarefas domésticas" e "recursos financeiros", os quais foram os mais apontados como barreiras. Nesse caso, seria importante dispor de maiores informações a respeito do tipo de atividade realizada pelos servidores administrativos no trabalho, o que possibilitaria uma melhor compreensão mais aprofundada desta situação.

Por se tratar de estudo transversal, realizado em população específica de servidores de uma determinada universidade, torna limitada a extensão dos resultados para trabalhadores em geral. E também por ser um estudo transversal não permite identificar relações de causalidade entre as variáveis.

Esses dados irão contribuir para a melhoria de saúde dos servidores, onde através dos dados referentes ao nível de atividade física e as barreiras encontradas para a sua prática, poderão servir de parâmetro comparativo para próximas investigações realizadas com esses servidores.

\section{CONCLUSÃO}

Conclui-se que as principais barreiras para a prática de AF percebidas pelos servidores foram, a jornada de trabalho extensa e a falta de energia. As barreiras do domínio social estiveram associadas à inatividade física, para ambos os gêneros.

Sendo assim, recomenda-se o desenvolvimento de políticas públicas saudáveis com o propósito de aumentar o estímulo a prática de $\mathrm{AF}$ e diminuir os fatores de risco para o surgimento de DCNT. 


\section{REFERÊNCIAS}

1. Barros MVG, Nahas MV. Comportamentos de risco, auto-avaliação do nível de saúde e percepção de estresse entre trabalhadores da indústria. Rev Saúde Pública. 2001; 35(6):554-563.

2. Silva SG, Silva MC, Nahas MV, Viana SL. Variables associates with leisuretime physical inactivity and main barriers to exercise among industrial workers in Southern Brazil. Cad Saúde Pública. 2011; 27(2):249-259.

3. Robroek, SJW, Van Den Berg TIJ, Plat JF. The role obesity and lifestyle behaviors in a productive workforce. Occup Environ Med 2011; 68:134-139.

4. Felipe-de-Melo ERT, Silva RCR, Assis AMO, Pinto EJ. Fatores associados à syndrome metabólica em trabalhadores administrativos de uma indústria de petróleo. Ciênc Saúde Coletiva. 2011; 16(8):3443-3452.

5. Hallal PC, Anderson LB, Bull FFC, Guthold R, Haskell W, Ekelund U. Global physical activity levels: Surveillance progress, pitfalls, and prospects. Lancet. 2012; 380(9838):247-257.

6. Gonçalves ECA, Rinaldi W, Nunes HEG, Capdeboscq MC, Silva DAS. Baixos niveis de atividade fisica em servidores públicos do sul do Brasil: associação com fatores sociodemográficos, hipercolesterolemia e diabetes. Rev Andal Med Deporte. 2017; 10(2):54-59.

7. Rocha SV, Pie ACS, Cardoso JP, Amorim CR, Carneiro LRV, Vilela ABA. Nivel de atividade fisica entre funcionários de uma instituição de ensino superior da Bahia. Ulbra Mov. 2011; 2(1):16-29.

8. Azevedo SF, Lopes AS. Atividade física desempenhada por trabalhadores brasileiros: uma revisão sistemática. Pensar Prát. 2012: 15(3):518-520.

9. Savio KEO. Sexo, renda e escolaridade associados ao nivel de atividade física de trabalhadores. Rev Saúde Pública. 2008; 42(3):457-63.

10. Malta DC, Silva JB Jr. Strategic Action Plan to Combat Non-Communicable Diseases in Brazil after three years of implementation, 2011-2013. Epidemiol Serv Saúde. 2014; 23(3):389-395.

11. Matsudo $S$, Araújo $T$, Matsudo $V$, Andrade $D$, Andrade $E$, Oliveira LC. Questionário Internacional de Atividade Física (IPAQ): estudo de validade e reprodutibilidade no Brasil. Rev Bras Ativ Fis Saúde. 2001; 6(2):5-18.

12. Martins MO, Petroski EL. Mensuração da percepção de barreiras para a prática de atividades físicas: uma proposta de instrumento. Rev Bras Cineantropom Desempenho Hum. 2000; 2(1):58-65.

13. Jesus GM, Jesus EFA. Nivel de atividade física e barreiras percebidas para a prática de atividades físicas entre policiais militares. Rev Bras Ciênc Esporte. 2012; 34(2):433-48.

14. Boscatto EC, Duarte MFS, Gomes MA. Estágios de mudança de comportamento e barreiras para a atividade fisica em obesos mórbidos. Rev Bras Cineantropom Desempenho Hum. 2011; 13(5):329-34.

15. World Health Organization (WHO). Obesity: preventing and managing the global epidemic. Geneva: World Health Organization; 2000.

16. De Sá-Silva SP, Yokoo EM, Salles-Costa R. Fatores demográficos e hábitos de vida relacionados com a inatividade física de lazer entre gêneros. Rev Nutr. 2013; 26(6):633-645.

17. Tucker JM, Tucker LA, Lecheminant J, Bailey B. Obesity increases risk of declining physical activity over time in women: A prospective cohort study. Obesity. 2013; 21(12):715-720.

18. Seck SM, Guéye S, Tamba K, Ba I. Prevalence of chronic cardiovascular and metabolic diseases in senegalese workers. A cross-sectional study, 2010. Prev Chronic Dis. 2013; 10:1-7.

19. Vargas LM, Pilatti LA, Gutiérrez GL. Inatividade fisica e fatores associados: um estudo com trabalhadores do setor metalomecânico do município de Ponta Grossa-PR. Rev Bras Ativ Fis Saúde. 2013; 18(1):32-42.

20. Florindo AA, Guimarães W, Cesar CLG, Barros MBA, Alves MCGP, Goldbaum M. Epidemiology of leisure, transportation, occupational, and household physical activity: prevalence and associated factors. J Phys Act Health. 2009; 6(5):625-632.
21. Duca GFD, Nahas MV, Garcia LMT, Mota J, Hallal PC, Peres MA. Prevalence and sociodemographic correlates of all domains of physical activity in Brazilian adults. Prev Med. 2013; 56(2):99-102.

22. Pinho PS, Araújo TM. Associação entre sobrecarga doméstica e transtornos mentais comuns em mulheres. Rev Bras Epidemiol. 2012;15(3):560-572.

23. Silva KS, Nahas MV, Peres KG, Lopes AS. Fatores associados à atividade física, comportamento sedentário e participação na Educação Física em estudantes do Ensino Médio em Santa Catarina, Brasil. Cad Saúde Pública. 2009; 25(10):2187-2200.

24. Proper $\mathrm{KI}$, Hildebrandt VH. Physical activity among dutch workers: Differences between occupations. Prev Med. 2006; 43:42-45

25. Bicalho PG, Hallal PC, Gazzineli A. Atividade fisica e fatores associados em adultos de area rural em Minas Gerais, Brasil. Rev Saúde Pública. 2010; 5(44):884-893.

26. Duncan MJ, Badland HM, Mummery WK. Physical activity levels by occupational category in non-metropolitan Australian adults. J Phys Act Health. 2010; 7:718-723.

27. Sousa TF, Nahas MV, Silva DAS, Del Duca GF, Peres MA. Fatores associados à obesidade central em adultos de Florianópolis, Santa Catarina: estudo de base populacional. Rev Bras Epidemiol. 2011; 14(2):296-309.

28. Brasil. Ministério da Saúde. VIGITEL 2014: Vigilância de fatores de risco e proteção para doenças crônicas por inquérito telefônico. Brasilia: MS; 2015.

29. Berria J, Petroski EL, Minatto G. Excesso de peso, obesidade abdominal e fatores associados em servidores de uma Universidade Federal Brasileira. Rev Bras Cineantropom Desempenho Hum. 2013, 15(5):535-550 537.

30. Fonseca MdJMd, Faerstein E, Chor D, Lopes CS, Andreozzi VL. Associações entre escolaridade, renda e Índice de Massa Corporal em funcionários de uma universidade no Rio de Janeiro, Brasil: estudo Pró-Saúde. Cad Saúde Pública. 2006; 22(11):2359-2367.

31. Martinez MC, Latorre MRDO. Fatores de risco para hipertensão arterial e diabetes melito em trabalhadores de empresa metalúrgica e siderúrgica. Arq Brasil Cardiol. 2016; 87:471-479.

32. Oliveira RAR, Moreira OC, Neto FA, Amorim W, Costa EG, Marins JCB. Prevalência de sobrepeso e obesidade em professores da Universidade Federal de Viçosa. Fisioter Mov Curitiba. 2011; 24:603-612.

33. Palacios C, Perez CM, Guzman M, Ortiz AP, Ayala A, Suarez E. Association between adiposity indices and cardiometabolic risk factors among adults living in Puerto Rico. Public Health Nutr. 2011; 14(10):1714-1723.

34. Ahmed F, Waslien C, Al-Sumaie MA, Prakash P. Secular trends and risk factors of overweight and obesity among Kuwaiti adults: National Nutrition Surveillance System data from 1998 to 2009. Public Health Nutr. 2012; 15(11):2124-2130.

35. Flegal KM, Carroll MD, Ogden CL, Curtin LR. Prevalence and trends in obesity among US adults, 1999-2008. JAMA Intern Med. 2010; 303(3):235241.

36. Rostambeigi N, Shaw JE, Atkins RC, Ghanbarian A, Cameron AJ, Forbes A, et al. Waist circumference has heterogeneous impact on development of diabetes in different populations: longitudinal comparative study between Australia and Iran. Diabetes Res Clin Pr. 2010; 88(1):117-24.

37. Tahara AK, Schwartz GM, Silva KA. Aderência e manutenção da prática de exercicios em academias. R Bras Ci e Mov. 2003; 11(4):7-12.

38. Mauro M, Taylor V, Wharton S, Sharma A. Barriers to obesity treatment. Eur J Intern Med. 2008; 19(3):173-80.

39. MartinsM, Petroski EL. Mensuração da percepção de barreiras para a prática de atividade física: uma proposta de instrumento. Rev Bras Cineantropom Desempenho Hum. 2000; 2(1): 58-65.

40. Reichert FF. The role of perceived personal barriers to engagement in leisuretime physical activity. Am J Public Health. 2007; 97(3):515-519. 